\title{
Initial Dynamical Evolution of Star Clusters with Tidal Field
}

\author{
So-Myoung Park ${ }^{1}$, Simon P. Goodwin ${ }^{2}$ and Sungsoo S. Kim ${ }^{1,3}$ \\ ${ }^{1}$ School of Space Research, Kyung Hee University, \\ 1732, Deogyeong-daero, Yongin-si 446-701, South Korea \\ email: smpark12@khu.ac.kr \\ ${ }^{2}$ Dept. of Physics \& Astronomy, University of Sheffield, \\ Sheffield S3 7RH, United Kingdom \\ email: s.goodwin@sheffield.ac.uk \\ ${ }^{3}$ Dept. of Astronomy \& Space Science, Kyung Hee University, \\ 1732, Deogyeong-daero, Yongin-si 446-701, South Korea \\ email: sungsoo.kim@khu.ac.kr
}

\begin{abstract}
Observations have been suggested that star clusters could form from the rapid collapse and violent relaxation of substructured distributions. We investigate the collapse of fractal stellar distributions in no, weak, and very strong tidal fields. We find that the rapid collapse of substructure into spherical clusters happens quickly with no or a weak tidal field, but very strong tidal fields prevent a cluster forming. However, we also find that dense Plummer spheres are also rapidly destroyed in strong tidal fields. We suggest that this is why the low-mass star clusters cannot survive near the galactic centre which has strong tidal field.
\end{abstract}

Keywords. Galaxy: open clusters and associations: general, Galaxy: center, Galaxy:kinematics and dynamics, methods: n-body simulations

\section{Introduction}

How star clusters form is one of the important questions in star formation. Observations show that many young stars are in dense clusters (Lada \& Lada 2003), even in regions where the tidal field is strong, e.g. the Arches and Quintuplet clusters, near the Galactic centre. Gas-rich star forming regions, however, are often found to be clumpy, filamentary and substructured (Cartwright \& Whitworth 2004, Konyves et al. 2010). Numerical simulations have shown that star clusters can form from initial fractal distributions with no tidal field (Parker et al. 2014 and references therein). But do dense nuclear star clusters like the Arches have to form at high densities, or can they collapse from substructure? Here we present the first results of simulations of how tidal fields can affect the initial evolution of initially clumpy star clusters. We concentrate on the evolution of low-mass $\left(<1000 \mathrm{M}_{\odot}\right)$ clusters in this short contribution.

\section{Method \& Initial Conditions}

Method. We use the Nbody6 code as modified by Kim (Kim et al. 2000). Tidal fields are applied at $30 \mathrm{pc}$ from the Galactic centre with the enclosed mass determined from Launhardt et al. (2002).

Initial conditions. We use initially fractal (Goodwin \& Whitworth 2004) and Plummer (1911) distributions. The number of stars is 999 and the mass of star cluster is about $700 \mathrm{M}_{\odot}$. Initial mass function follows Macshberger (2013). In a paper to follow we also 

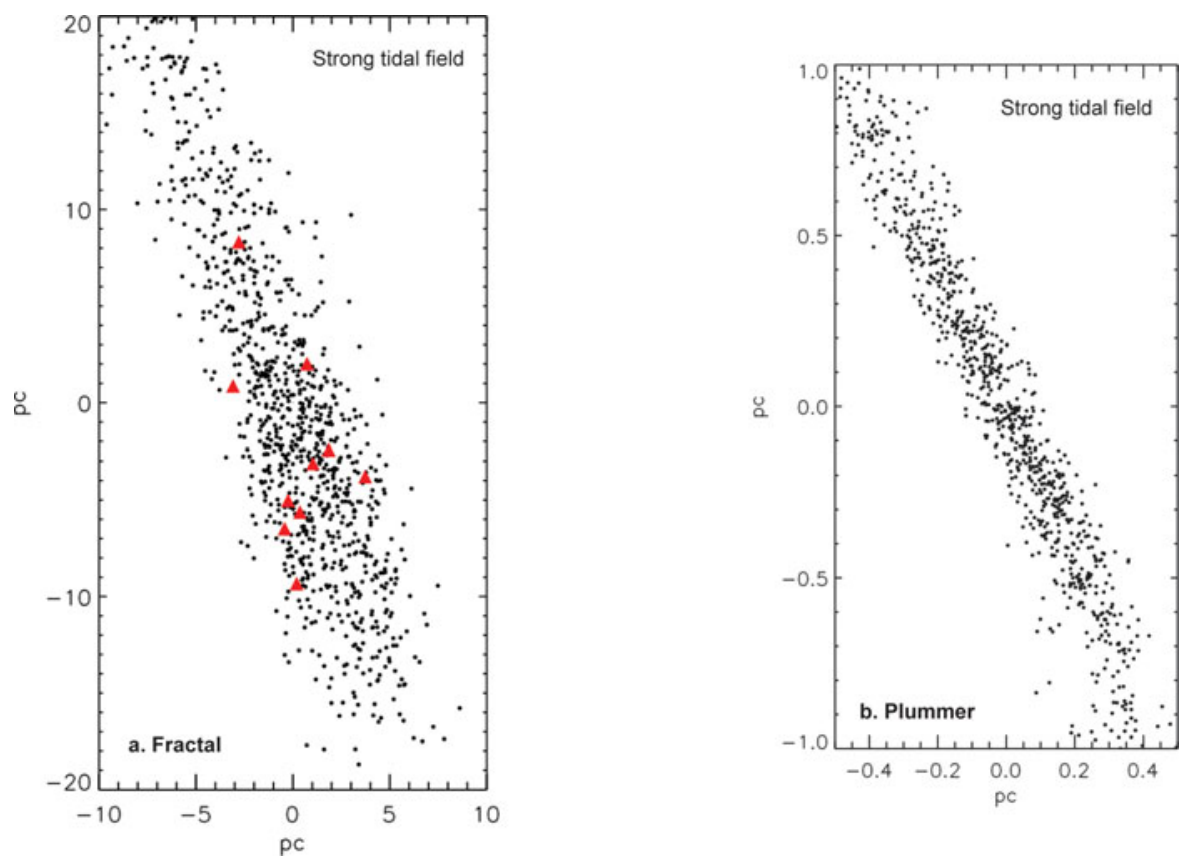

Figure 1. The morphologies of star clusters at $2 \mathrm{Myr}$, in panel (a) starting with an initially fractal distribution, and in panel (b) starting with a dense Plummer sphere.

examine different strong tidal fields at $100 \mathrm{pc}$ and $200 \mathrm{pc}$, as well as the effect of weak (Solar radius) tidal fields.

\section{Results \& Conclusion}

We find that a low-mass initially fractal distribution can collapse and form a dense, spherical cluster within $2 \mathrm{Myr}$ with no tidal field (or a weak tidal field). In a strong (30 pc from the Galactic centre) tidal field, however, collapsing clusters are ripped apart within 2 Myr. However, simulations of dense low-mass Plummer spheres in a strong tidal field are also shredded within $2 \mathrm{Myr}$, despite their initial size being smaller than their tidal radii. Fig. 1 shows the state of both the initially fractal (left panel), and Plummer (right panel) distributions after $2 \mathrm{Myr}$. This means that low-mass star clusters cannot survive near the Galactic Centre, no matter what their initial conditions are.

\section{References}

Cartwright, A. \& Whitworth, A. P. 2004, MNRAS, 348, 589

Goodwin, S. P. \& Whitworth, A. P. 2004, A\& $A$, 413, 929

Kim, S. S., Figer, Donald F., Lee, Hyung Mok, et al. 2000, ApJ, 545, 301

Konyves, V., Andre, Ph., Menshchikov, A., et al. 2010, A\&\&A, 518, L106

Lada, C. J. \& Lada, E. A. 1993, ARA\&A, 41, 57

Launhardt, R., Zylkz, R., \& Mezger, P. G. 2002, A\& A, 384, 112

Maschberger, T. 2013, MNRAS, 429, 1725

Parker, R. J., Wright, N. J., Goodwin, S. P., et al. 2014, MNRAS, 438, 620

Plummer, H. C. 1911, MNRAS, 71, 460 